\section{Aurikularanhänge und ihre Bedeutung für die Dermatologie}

\author{
T. Jansen ${ }^{1}$, R. Romiti ${ }^{2}$, Ulrike Memmel ${ }^{1}$, P. Altmeyer ${ }^{1}$ \\ ${ }^{1}$ Klinik für Dermatologie und Allergologie der Ruhr-Universität \\ Bochum (Direktor: Prof. Dr. med. P. Altmeyer) \\ 2 Universität von São Paulo, Brasilien \\ (Direktor: Prof. Dr. med. E. Rivitti)
}

\begin{abstract}
Zusammenfassung. Aurikularanhänge sind seltene Fehlbildungen des äußeren Ohres, die aus dem ersten Kiemenbogen hervorgehen. Es handelt sich um asymptomatische hautfarbene Knoten, die am häufigsten in der Präaurikularregion vorkommen. Eine familiäre Häufung dieser Veränderungen wurde ebenso beschrieben wie ihr Auftreten als Teilsymptom von Syndromen. Beim Goldenhar-Syndrom werden derartige Fehlbildungen regelmäßig angetroffen. Da Aurikularanhänge mit anderen Strukturen wie Epidermoidzysten verwechselt werden können, haben sie für den Arzt eine Bedeutung für die Differenzialdiagnose von Ohrfehlbildungen. Die Therapie besteht in der einfachen Exzision.
\end{abstract}

Accessory Tragi and their Importance for Dermatology. Accessory tragus is a rare congenital malformation of the external ear, originating in the first branchial arch. Most frequent localization of the asymptomatic skin-colored lesion is the preauricular region. Familial occurrence of accessory tragus as well as association with a variety of developmental abnormalities of the first and second branchial arch have been reported. The only disorder with which accessory tragus is consistently found is Goldenhar syndrome. Accessory tragus may be confused with other structures such as an epidermoid cyst, therefore this congenital malformation is important for the differential diagnosis of ear lesions. Simple excision is the treatment of choice.

\section{Einleitung}

Mit Ausnahme des Tragus, der sich vom ersten Kiemenbogen ableitet, entwickelt sich die Ohrmuschel aus dem zweiten Kiemenbogen. Aurikularanhänge sind seltene Fehlbildungen des äußeren Ohres. In der Mehrzahl der Fälle treten sie als isolierte Fehlbildungen in Erscheinung, jedoch ist ein kombiniertes Auftreten mit anderen Missbildungen des ersten und zweiten Kiemenbogens möglich.

Akt Dermatol 2001; 27: 233-235

(c) Georg Thieme Verlag Stuttgart · New York ISSN 0340-2541

\section{Embryologie des äußeren Ohres}

Am Ende der fünften Embryonalwoche beginnt die Entwicklung des äußeren Ohres aus einer Reihe von Mesenchymverdichtungen, die an den dorsalen Enden des ersten und zweiten Kiemenbogens (Mandibular- oder Hyoidbogen) auftreten und die erste Kiemenfurche umgeben [33]. In der sechsten Embryonalwoche treten an jedem Bogen drei kleine Vorwölbungen (Ohrmuschel- oder Aurikularhöcker) auf. Diese bewegen sich nach dorsal, verschmelzen miteinander und bilden die Ohrmuschel. Entlang dieser Migrationslinie können sich Aurikularanhänge entwickeln. Aus dem ersten Aurikularhöcker entsteht der Tragus, aus dem zweiten und dritten formen sich die Ohrhelix und die Cymba conchae. Aus dem vierten und fünften Aurikularhöcker bildet sich die Concha. Der sechste Aurikularhöcker schließlich formt den Antitragus. Am Ende der sechsten Embryonalwoche besteht das äußere Ohr zu nahezu gleichen Anteilen aus dem ersten und zweiten Kiemenbogen. In den folgenden zwei Wochen der Embryonalentwicklung bildet sich das Mesenchym des ersten Kiemenbogens zurück. Zum Zeitpunkt der Geburt hat der erste Kiemenbogen lediglich einen Anteil von etwa $15 \%$ am äußeren Ohr. Die übrigen $85 \%$ resultieren aus dem zweiten Kiemenbogen. Die erste Kiemenspalte, welche zwischen dem ersten und zweiten Kiemenbogen liegt, vertieft sich und formt schließlich den äußeren Gehörgang. Das Ektoderm der ersten Kiemenfurche und das Endoderm der ersten Schlundtasche, welches das Mittelohr auskleidet, grenzen im Trommelfell aneinander. Das zwischen beiden Epithelschichten liegende Gewebe stammt vom Mesoderm ab und entwickelt sich schließlich zur fibrösen Mittelschicht des Trommelfells. Um den dritten Lebensmonat beginnt die Ossifikation vom Randbereich der Membrana tympani unter Bildung des tympanischen Ringes.

\section{Kasuistik}

Bei einem jetzt dreijährigen Mädchen bestanden seit der Geburt mehrere asymptomatische, bis zu $2 \times 3 \mathrm{~mm}$ große, hautfarbene Knoten mit glatter, haarloser Oberfläche auf dem Tragus des rechten Ohres, die seitdem mehr oder weniger unverändert geblieben waren (Abb.1). Die Knoten fühlten sich derb an, wobei die darüberliegende Haut nicht oder kaum verschieblich war. Die Ohrmuschel war normal geformt. Es fanden sich keine weiteren Fehlbildungen. Die allgemeine körperliche und geistige Entwicklung war altersentsprechend normal. In der Familie fanden sich keine Hinweise auf entwick- 


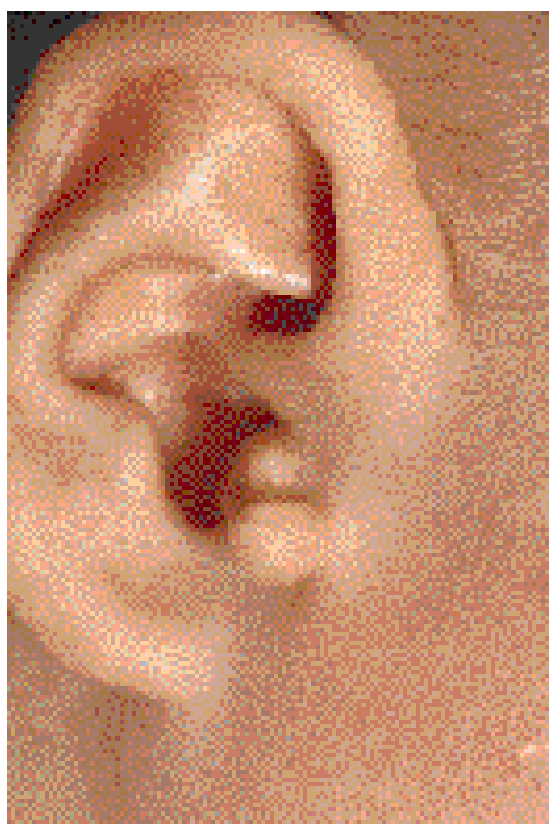

Abb. 1 Mehrere hautfarbene Knoten auf dem Tragus des rechten Ohres. lungsbedingte oder kutane Fehlbildungen. Schwangerschaft und Geburt waren komplikationslos verlaufen. Auf Wunsch der Eltern wurde von einer Exzision der Veränderungen abgesehen.

\section{Diskussion}

Aurikularanhänge werden in der Literatur als überzählige Ohrmuschel $[5,15,28,29]$, branchiogener Knorpelnävus [25], akzessorisches äußeres Ohr [3], Hautpapille [11] oder Polyotie [18] geführt. Es handelt sich um Fehlbildungen des ersten Kiemenbogens, da der Tragus als einzige Struktur des äußeren Ohres aus dem ersten Kiemenbogen hervorgeht.

Die Prävalenz von Aurikularanhängen als isolierte Fehlbildungen wird auf etwa $1,7: 1000$ geschätzt $[16,20]$. Bilaterales Auftreten wird in $6 \%$ der Fälle beobachtet, was einer Prävalenz von 9-10: 100000 entspricht [25,32]. Guszman [9] fand in einem Kollektiv von 11000 Patienten lediglich bei einem Patienten einen bilateral ausgebildeten Aurikularanhang. Dieses Ergebnis steht in Einklang mit den zuvor genannten Angaben zur Prävalenz.

Aurikularanhänge manifestieren sich als weiche oder derbe, hautfarbene Knoten entlang einer gedachten Linie zwischen Tragus und Mundwinkel oder entlang des vorderen Randes des Musculus sternocleidomastoideus [12]. Sie können aber auch direkt auf dem Tragus entstehen. Diese Fehlbildungen sind vermutlich bereits bei der Geburt vorhanden, bleiben aber oftmals für eine gewisse Zeit unbemerkt. Meist entstehen Aurikularanhänge als solitäre Gebilde in der Präaurikularregion vor dem Tragus. Über das Auftreten multipler Veränderungen wurde ebenso berichtet wie über die Lokalisation an Wangen, lateralen Halspartien [2], Glabella [24] oder Suprasternalregion. Eine familiäre Häufung von Aurikularanhängen wurde beschrieben, wobei in diesen Fällen eine auffallende Übereinstimmung in der anatomischen Lage innerhalb einer betroffenen Familie vorhanden war $[11,25,30]$. In der Regel handelt es sich um geringfügige kongenitale Fehlbildungen, die jedoch in manchen Fällen zu einer kosmetischen Beeinträchtigung führen können.

In einer retrospektiven Studie an 55 Patienten mit Aurikularanhängen wurde lediglich in $20 \%$ der Fälle eine korrekte klinische Diagnose gestellt [4]. Die am häufigsten genannten Fehldiagnosen waren Hautanhängsel, Polypen, Papillome oder Fibrome. Histopathologisch wurde dagegen in weniger als der Hälfte der Fälle eine korrekte Diagnose gestellt.

Aurikularanhänge können mit einer Reihe von entwicklungsbedingten Fehlbildungen des ersten und zweiten Kiemenbogens vergesellschaftet sein, die aus Tab. 1 ersichtlich sind $[6,7,21]$. Bei unserer Patientin war jedoch keine Assoziation mit anderen Fehlbildungen nachweisbar. In einer Studie, die sich mit assoziierten Fehlbildungen der Kiemenbögen befasste, fanden sich bei 3 von 52 Patienten (5,8\%) Aurikularanhänge [4]. Mit Aurikularanhängen assoziierte Erkrankungen sind die Dysostosis mandibulofacialis (Treacher-Collins-Syndrom) in vollständiger oder partieller Ausprägung [17] und das Goldenhar-Syndrom (Okulo-aurikulo-vertebrales Syndrom). Dieses beinhaltet neben Aurikularanhängen die Hypoplasie einer Gesichtshälfte, epibulbär lokalisierte Dermoidzysten, Kolobome des Augenlides oder der Iris, Mittelohrdefekte mit Schwerhörigkeit sowie Wirbelkörperdefekte [26]. Das Goldenhar-Syndrom ist wahrscheinlich das einzige Syndrom, bei dem Aurikularanhänge als konstantes Merkmal angetroffen werden [21]. Beim 4p-Syndrom (Wolf-Hirschhorn-Syndrom), das mit partieller Deletion des kurzen Arms von Chromosom 4 einhergeht [10], finden sich Aurikularanhänge ebenso wie beim TownesBrocks-Syndrom [19] und VACTERL-Syndrom (gelegentlich auch als VATER-Syndrom bezeichnet) [14,31]. Die beiden letztgenannten Syndrome sind durch kongenitale Fehlbildungen von Anus, Ohr, Nieren und Radius gekennzeichnet. Komplexe Fehlbildungen im Bereich der Harnblase und der ableitenden Harnwege sollten zur Suche nach assoziierten Anomalien wie Aurikularanhängen im Rahmen des VACTERL-Syndroms (VACTERL: V, vertebrale und vaskuläre Fehlbildungen; A, Analatresie, Aurikularanhänge; C, kardiovaskuläre Fehlbildungen; T, tracheoösophageale Fehlbildungen; E, Ösophagusatresie; R, renale, radiale und Rippenfehlbildungen; L, Extremitätenfehlbildungen) Anlass geben. In einem Fallbericht fanden sich $\mathrm{Au}-$ rikularanhänge bei einem Patienten mit kutaner Muzinose, Entwicklungsverzögerung, kongenitalem Katarakt und bilateraler Leistenhernie [27]. Da Aurikularanhänge auf eine assoziierte Schwerhörigkeit hinweisen können, wird die Durchführung einer Audiometrie bei allen Patienten mit derartigen Fehlbildungen empfohlen.

Tab.1 Mit Aurikularanhängen assoziierte Syndrome

\begin{tabular}{lll}
\hline Syndrom & Erbgang & Aurikularanhänge \\
\hline Goldenhar-Syndrom & autosomal-rezessiv & regelmäßig \\
Townes-Brocks-Syndrom & autosomal-dominant & selten \\
Treacher-Collins-Syndrom & autosomal-dominant & selten \\
VACTERL-Syndrom & autosomal-dominant & selten \\
Wolf-Hirschhorn-Syndrom & autosomal-dominant & selten \\
\hline
\end{tabular}


Histopathologisch bestehen Aurikularanhänge aus einem schmalen Stratum corneum, durchsetzt von unregelmäßig angeordneten Vellushaarfollikeln [23]. Meist sind ekkrine Schweißdrüsen vorhanden. Das Stroma enthält Ansammlungen von reifem Fettgewebe. Eine zentrale Platte aus elastischem Knorpel besteht in der Mehrzahl der Fälle. Gelegentlich wurden geringe Anteile von Skelettmuskulatur gefunden [4]. In manchen Fällen lassen sich gleichzeitig histopathologische Befunde von Aurikularanhängen und Haarfollikelnävi nachweisen. Aus diesem Grund wurde angenommen, dass Haarfollikelnävi inkomplette Aurikularanhänge mit einem geringen Anteil an Fettzellen darstellen [1]. Das klinische und histopathologische Erscheinungsbild von Aurikularanhängen ist vergleichbar mit dem kongenitaler Mittellinienhamartome, allerdings besitzen letztere keine zentrale Platte aus elastischem Knorpel, zudem ist der Anteil quergestreifter Muskelfasern deutlich höher [8]. Darüber hinaus sind Aurikularanhänge im Gegensatz zu Mittellinienhamartomen praktisch nie in der Kinnmitte lokalisiert.

Falls kosmetisch beeinträchtigend, können Aurikularanhänge exzidiert werden. Die Ergebnisse sind gut, wenn alle Knorpelanteile entfernt werden, die bis tief in die Subkutis hineinreichen können [24]. Wird lediglich eine oberflächliche Exzision durchgeführt, können unvollständig entfernte Knorpelanteile die Wundheilung beeinträchtigen. Selbst wenn der Wundheilungsprozess regelrecht verläuft, können spitze Knorpelanteile zu einer Chondrodermatitis führen.

\section{Literatur}

${ }^{1}$ Ban M, Kamiya H, Yamada T, Kitajima Y. Hair follicle nevi and accessory tragi: variable quantity of adipose tissue in connective tissue framework. Pediatr Dermatol 1997; 14: 433-436

2 Birkett J. Congenital supernumerary and imperfectly developed auricles on the sides of the neck. Trans Pathol Soc London 1858; 9: $448-449$

${ }^{3}$ Bose P. Accessory external ear. Indian Med Gaz 1922; 57: 139 140

${ }^{4}$ Brownstein MH, Wanger N, Helwig EB. Accessory tragi. Arch Dermatol 1971; 104: 625-631

${ }^{5}$ Clarke WB. Supernumerary auricles. Illus Med News 1888; 1: 321

${ }^{6}$ Cohen PR, Gilbert-Barness E. Pathological cases of the month: accessory tragus. Am J Dis Child 1993; 147: 1123-1124

${ }^{7}$ Cosman BC. Bilateral accessory tragus. Cutis 1993; 51: 199-200

${ }^{8}$ Elgart GW, Patterson JW. Congenital midline hamartoma: case report with histochemical and immunohistochemical findings. Pediatr Dermatol 1990; 7: 199-201

${ }^{9}$ Guszman J. Beiträge zur Lehre der branchiogenen Ohr- und Halsanhänge. Z Ges Anat 1926; 81: 554-562

10 Guthrie RD, Aase JM, Asper AC, Smith DW. The 4p-syndrome: a clinically recognizable chromosomal deletion syndrome. Am J Dis Child 1971; 122: 421 - 425

11 Jenkins R. The occurrence of a skin papillus through four human generations. J Hered 1928; 19: 174

12 Kaufmann R. Aurikularanhänge in der Differentialdiagnose zervikaler Tumoren. Akt Dermatol 1987; 13: $63-65$

${ }^{13}$ Kim SW, Moon SE, Kim JA. Bilateral accessory tragi on the suprasternal region. J Dermatol 1997; 24: 543 - 545

${ }^{14}$ Kurnit DM, Steele MW, Pinsky L, Dibbins A. Autosomal dominant transmission of a syndrome of anal, ear, renal, and radial congenital malformations. J Pediatr 1978; 93: 270-273

${ }^{15}$ Lake R. Double supernumerary auricle. Illus Med News 1888; 1 : 322
${ }^{16}$ Melnick M, Myrianthopoulos NC. External ear malformations: epidemiology, genetics, and natural history. Birth Defects 1979; 15: $1-138$

${ }^{17}$ Meyerson MD, Jensen KM, Meyers JM, Hall BD. Nager acrofacial dysostosis: early intervention and long-term planning. Cleft Palate J 1977; 14 : $35-40$

${ }^{18}$ Miller CS, Miller KF. Supernumerary ears: report of three cases. Arch Dermatol Syphilol 1949; 60: 601 -608

${ }^{19}$ Monteiro de Pina-Neto J. Phenotypic variability in Townes-Brocks syndrome. Am J Med Genet 1984; 18: 147-152

${ }^{20}$ Ostmann P. Die Missbildungen des äußeren Ohres unter den Volksschulkindern des Kreises Marburg. Arch Ohrheilk 1903; 58: $168-170$

${ }^{21}$ Resnick KI, Soltani K, Bernstein JE, Fathizedeh A. Accessory tragi and associated syndromes involving the first branchial arch. J Dermatol Surg Oncol 1981; 7: 39-41

${ }^{22}$ Satoh T, Tokura Y, Katsumata M, Sonoda T, Takigawa M. Histological diagnostic criteria for accessory tragi. J Cutan Pathol 1990; 17: 206-210

${ }^{23}$ Sayama S, Tagami H. Cartilaginous nevus on the glabella. Acta Derm Venereol (Stockh) 1982; 62: 180-181

${ }^{24}$ Sebben JE. The accessory tragus - no ordinary skin tag. J Dermatol Surg Oncol 1989; 15: 304-307

25 Siemens HW. Zur Kenntnis der sogenannten Ohr- und Halsanhänge (branchiogene Knorpelnaevi). Arch Dermatol Syphilol 1921; 132: 186-205

${ }^{26}$ Sohi AS, Sohi BK. Oculo-auriculo-vertebral syndrome (Goldenhar's syndrome). Int J Dermatol 1978; 4: 339-341

${ }^{27}$ Stokes KS, Rabinowitz LG, Segura AD, Esterly NB. Cutaneous mucinosis of infancy. Pediatr Dermatol 1994; 11: 246-251

${ }^{28}$ Sutton JB. On branchial fistulae, cysts, diverticula, and supernumerary auricles. J Anat Physiol 1887; 21: 289-298

${ }^{29}$ Sutton JB. Supernumerary auricles. Illus Med News 1888; 1: $320-321$

${ }^{30}$ Teja K, Cooper PH. Familial occurrence of accessory tragus. J Pediatr Surg 1981; 16: $725-726$

${ }^{31}$ Werner W, Beintker M, Schubert J, Kaiser WA. Das VATER-Syndrom aus urologischer Sicht. Urologe 1998; 37: 203 - 205

32 Wiechmann A. Bericht über die in den Jahren 1893 bis 1. Juli 1912 in der Königlichen Universitäts-Poliklinik für Ohren-, Nasen- und Halskrankheiten in Göttingen beobachteten angeborenen Missbildungen des Ohres. Göttingen: Dissertation, 1912

${ }^{33}$ Wood-Jones F, Chuan IW. The development of the external ear. J Anat 1933; 68: 525-533

\section{Dr. med. T. Jansen}

Klinik für Dermatologie und Allergologie

Ruhr-Universität Bochum

Gudrunstraße 56

44791 Bochum 